\title{
Dominant mutations of the Notch ligand Jagged1 cause peripheral neuropathy
}

\author{
Jeremy M. Sullivan, ${ }^{1}$ William W. Motley, ${ }^{1}$ Janel O. Johnson, ${ }^{2}$ William H. Aisenberg, ${ }^{1}$ Katherine L. Marshall,' Katy E.S. Barwick, ${ }^{3}$ \\ Lingling Kong, ${ }^{1}$ Jennifer S. Huh, ${ }^{1}$ Pamela C. Saavedra-Rivera, ${ }^{1}$ Meriel M. McEntagart, ${ }^{4}$ Marie-Helene Marion, ${ }^{5}$ Lucy A. Hicklin, ${ }^{6}$ \\ Hamid Modarres, ${ }^{5}$ Emma L. Baple, ${ }^{3}$ Mohamed H. Farah,, ${ }^{1,7}$ Aamir R. Zuberi, ${ }^{8}$ Cathleen M. Lutz, ${ }^{8}$ Rachelle Gaudet, ${ }^{9}$ \\ Bryan J. Traynor, ${ }^{2,10}$ Andrew H. Crosby, ${ }^{3}$ and Charlotte J. Sumner ${ }^{1,7}$ \\ 'Department of Neurology, Johns Hopkins University School of Medicine, Baltimore, Maryland, USA. '2aboratory of Neurogenetics, National Institute on Aging, NIH, Bethesda, Maryland, USA. ${ }^{3}$ RILD \\ Wellcome Wolfson Centre, Royal Devon and Exeter NHS Foundation Trust, Exeter, Devon, United Kingdom. Medical Genetics, Clinical Developmental Sciences, St. Ceorge's University of London, London, \\ United Kingdom. ${ }^{5}$ Department of Neurology and ${ }^{6}$ Department of Ears, Nose and Throat (ENT), St. Ceorge's Hospital, London, United Kingdom. ${ }^{7}$ Solomon H. Snyder Department of Neuroscience, Johns \\ Hopkins University School of Medicine, Baltimore, Maryland, USA. ${ }^{8}$ Cenetic Resource Science, The Jackson Laboratory, Bar Harbor, Maine, USA. ${ }^{9}$ Department of Molecular and Cellular Biology, Harvard \\ University, Cambridge, Massachusetts, USA. ${ }^{10}$ Brain Sciences Institute, Department of Neurology, Johns Hopkins Hospital, Baltimore, Maryland, USA.
}

\begin{abstract}
Notch signaling is a highly conserved intercellular pathway with tightly regulated and pleiotropic roles in normal tissue development and homeostasis. Dysregulated Notch signaling has also been implicated in human disease, including multiple forms of cancer, and represents an emerging therapeutic target. Successful development of such therapeutics requires a detailed understanding of potential on-target toxicities. Here, we identify autosomal dominant mutations of the canonical Notch ligand Jagged1 (or JAC1) as a cause of peripheral nerve disease in 2 unrelated families with the hereditary axonal neuropathy Charcot-Marie-Tooth disease type 2 (CMT2). Affected individuals in both families exhibited severe vocal fold paresis, a rare feature of peripheral nerve disease that can be life-threatening. Our studies of mutant protein posttranslational modification and localization indicated that the mutations (p.Ser577Arg, p.Ser650Pro) impair protein glycosylation and reduce JAG1 cell surface expression. Mice harboring heterozygous CMT2-associated mutations exhibited mild peripheral neuropathy, and homozygous expression resulted in embryonic lethality by midgestation. Together, our findings highlight a critical role for JAC1 in maintaining peripheral nerve integrity, particularly in the recurrent laryngeal nerve, and provide a basis for the evaluation of peripheral neuropathy as part of the clinical development of Notch pathway-modulating therapeutics.
\end{abstract}

\section{Introduction}

The Notch pathway is a highly conserved cell-cell signaling mechanism that functions in virtually all tissues to regulate multiple aspects of cell fate and homeostasis. Dysregulation of Notch signaling outputs has been shown to cause or contribute to several human diseases, including developmental syndromes and adult-onset diseases. Due to its role as an oncogenic driver in several forms of cancer, the Notch pathway is an emerging therapeutic target (1). The on-target toxicity and side effects

Authorship note: JMS and WWM are co-first authors.

Conflict of interest: WWM is currently an employee of Third Rock Ventures. J0 j is currently an employee of Quest Diagnostics. BJT has received grant support from Merck and Microsoft Research. BJT holds European Union (EP2751284A1, Method For Diagnosing A Neurodegenerative Disease) and US (20180187262) patents on clinical testing and therapeutic intervention for the hexanucleotide repeat expansion of the C9orf72 gene. CJS has received grant support from and holds patents (W02016/164896 "Modulation of SMN expression," W02017/21884 "Combinations for the Modulation of SMN expression") with lonis Pharmaceuticals; has served as a paid advisor, consultant, and/or speaker for Biogen, PTC Therapeutics, Roche/Genentech, AveXis, and Cytokinetics; and is an associate editor for the JCI. This arrangement has been approved by Johns Hopkins University in accordance with its conflict of interest policies. Copyright: @ 2020, American Society for Clinical Investigation.

Submitted: February 13, 2019; Accepted: December 12, 2019; Published: February 17, 2020 Reference information: / Clin Invest. 2020;130(3):1506-1512.

https://doi.org/10.1172/JCl128152. resulting from pan-Notch inhibition (1) have prompted the development of approaches targeting specific Notch pathway components $(1,2)$. Given the highly pleotropic roles of Notch signaling, however, continued identification of Notch-dependent tissues will play an important role in the development of such targeted approaches for clinical use.

Here, we show that autosomal dominant mutations in the canonical Notch ligand Jagged1 (or JAG1) are a cause of the peripheral nerve disease Charcot-Marie-Tooth disease type 2 (CMT2). Individuals harboring these mutations exhibit severe vocal fold paresis, a rare clinical feature of peripheral neuropathy (3) that can be life life-threatening. These findings identify a key role for JAG1 in maintaining peripheral nerve integrity and highlight potential on-target toxicities that may be associated with JAG1-targeted therapeutics.

\section{Results and Discussion}

We examined 2 unrelated CMT2 families with similar clinical features (Figure 1 and Supplemental Tables 1 and 2; supplemental material available online with this article; https:/doi. org/10.1172/JCI128152DS1). Vocal fold paresis was severe, often requiring tracheostomy (Figure $1 \mathrm{E}$ and Supplemental Table 1), whereas neuropathy of the limbs was mild (Supplemental Tables 1 and 2). Whole exome sequencing (WES) of 3 affected 
A

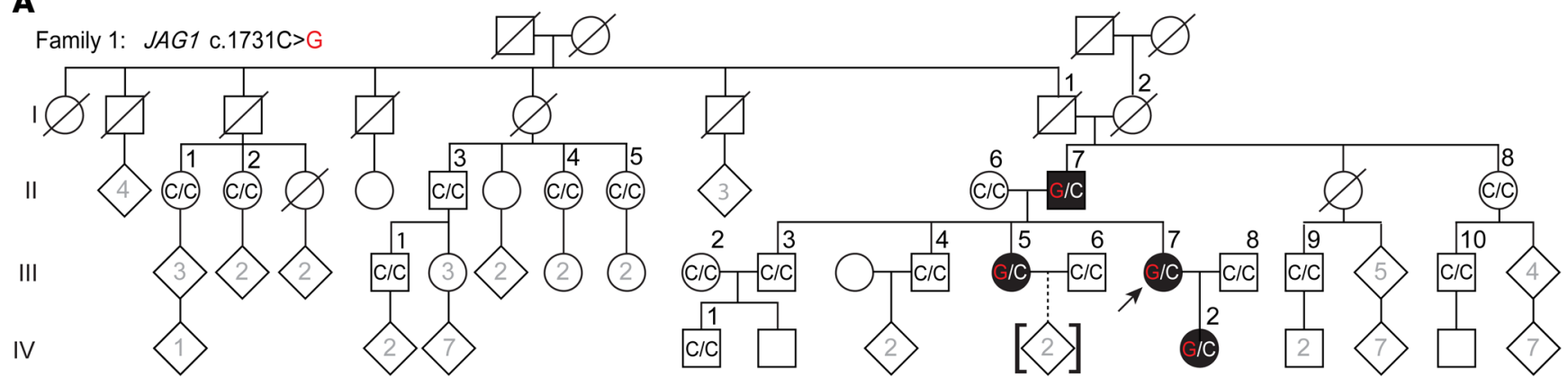

B

C
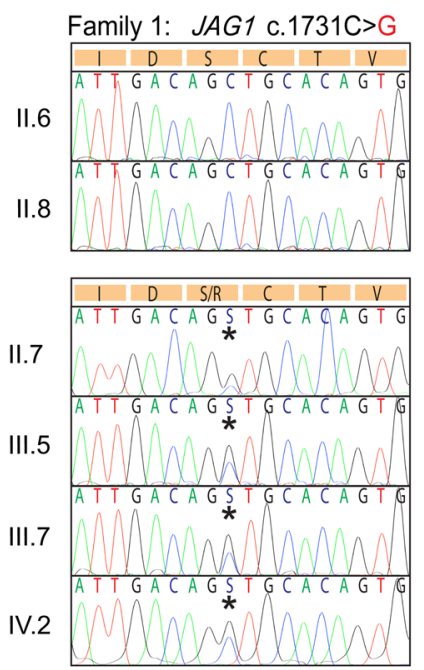
Family 1:

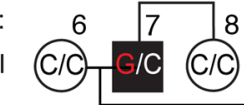

III
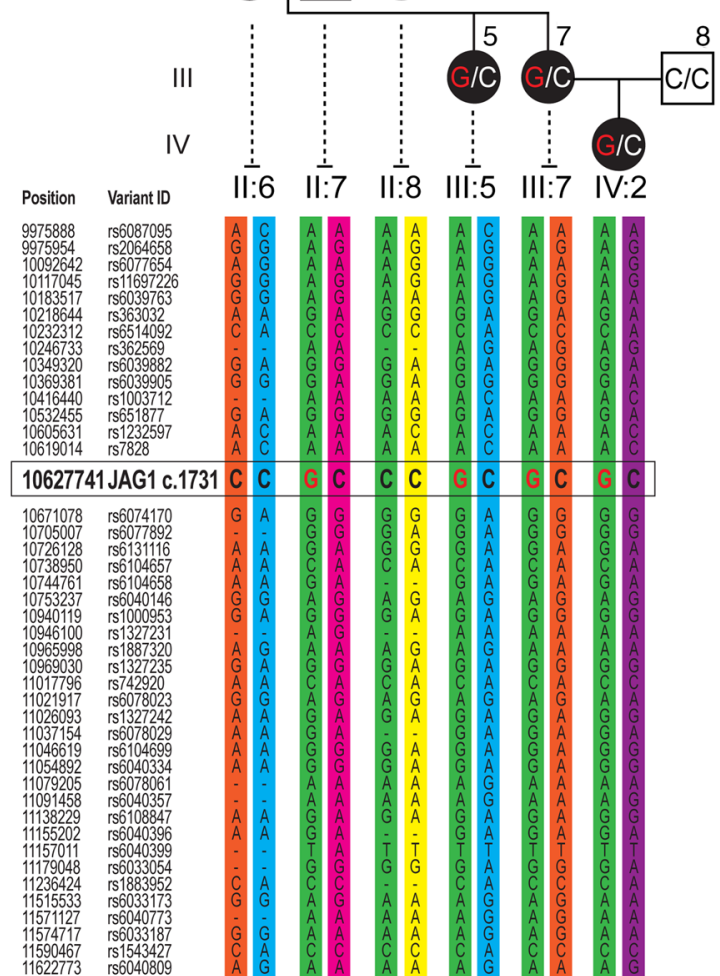

D Family 2: JAG1 c.1948T>C

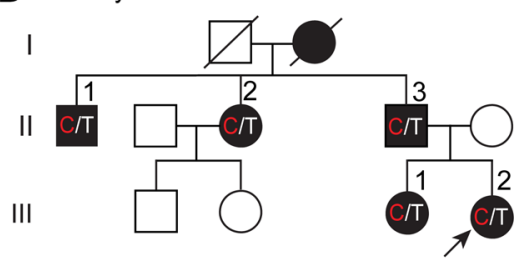

E
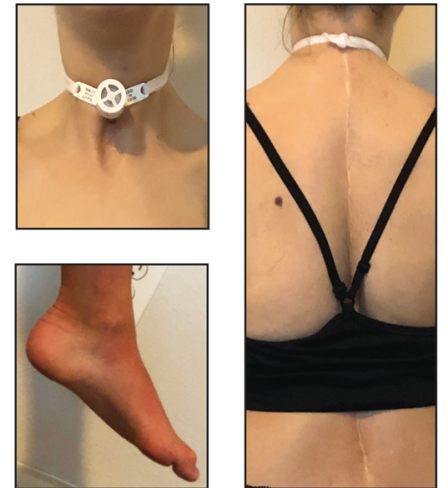

Figure 1. JAG1 mutations segregate with CMT2 and vocal fold paresis in $\mathbf{2}$ unrelated families. (A) Pedigree of family 1 demonstrating affected subjects in 3 generations (arrow indicates proband). The genotypes of individuals whose DNA was Sanger sequenced are shown. The parents of II.7 are deceased, but did not show any signs of peripheral neuropathy or vocal fold paresis. (B) Sanger sequencing of the JAG1 gene from unaffected (II.6 and II.8) and affected (II.7, III.5, III.7, IV.2) individuals from family 1, showing the heterozygous c.1731C >G variant (asterisks). (C) Haplotype analysis of family 1, with the CMT2associated haplotype shown in green. (D) Pedigree of family 2 demonstrating affected subjects across 4 generations. Family members whose DNA was examined have been numbered. (E) Photographs of individual IV.2 (family 1) illustrating her tracheostomy, scoliosis repaired with surgeries, and mild pes cavus (clockwise from top left).

individuals from family 1 (Figure 1A, individuals II.7, III.5, and III.7) did not identify variants in genes known to cause CMT. Of 15 candidate variants, only 1 segregated appropriately after Sanger sequencing of 14 additional affected and unaffected family members: a variant c. $1731 \mathrm{C}>\mathrm{G}$ in exon 14 of the JAG1 gene (Figure 1, A and B). We performed haplotype analysis to investigate the possibility that this mutation arose de novo in individual II.7, given his lack of affected parents and siblings. The disease-associated haplotype shared among all 4 affected individuals of family 1 (Figure 1C) was also shared by individ- ual II.8, a sibling of II.7. Importantly, however, individual II.8 did not harbor the $c .1731 \mathrm{C}>\mathrm{G}$ substitution, indicating that this variant likely arose de novo in II.7. WES of individual III. 2 from family 2 also did not identify variants in known neuropathyassociated genes (Figure 1D and Supplemental Tables 1 and 2), but did reveal a second variant in JAG1, c.1948T>C (exon 15), which cosegregated with the phenotype in all 5 affected family members from whom DNA was available (Figure 1D). Neither the c.1731C $>$ G variant (family 1) nor the c.1948T $>C$ variant (family 2) is present in the Genome Aggregation Database 

A H. sapiens
CMT2
P. troglodytes
B. taurus
R. norvegicus
M. musculus
G. gallus
X. tropicalis
$D$. rerio
D. melanogaster

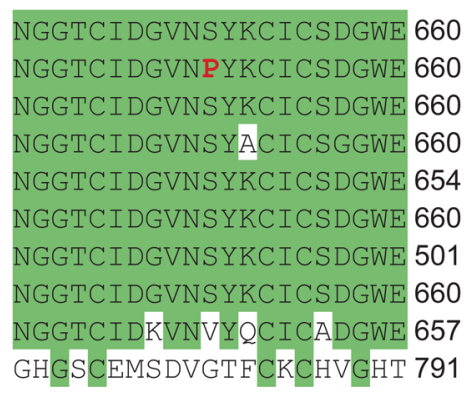

B
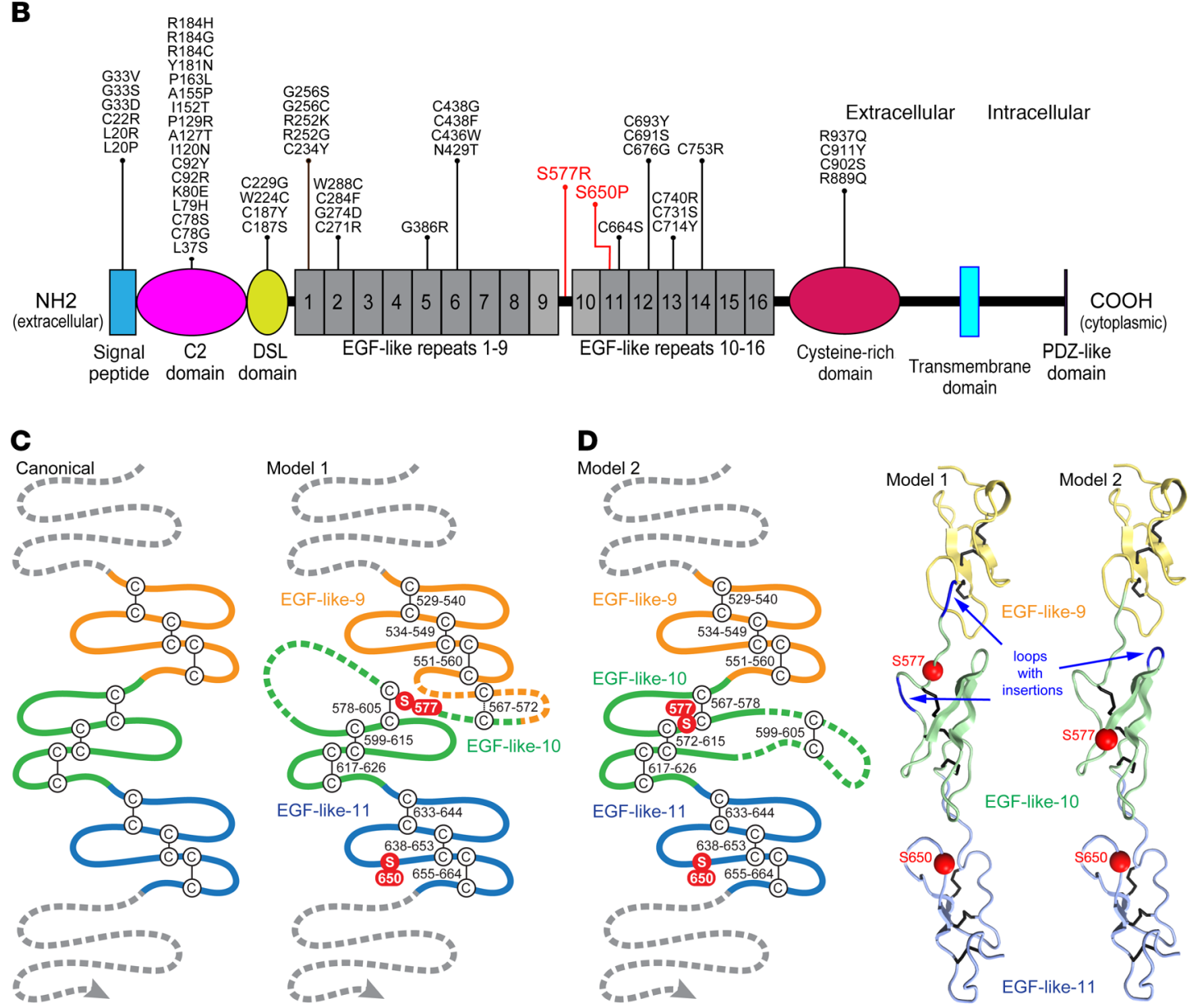

Figure 2. Neuropathy-associated mutations affect conserved amino acids in the extracellular domain of JAG1. (A) Clustal Omega alignments of the JAG1 protein from divergent species. The p.Ser577Arg and p.Ser650Pro mutations both disrupt serine residues located in highly conserved regions of JAG1. (B) The domain structure of JAG1, with the CMT2-associated mutations indicated in red. Though Alagille syndrome is primarily caused by JAG1 gene deletion or truncating mutations, a number of missense mutations (shown in black) have been described (see Supplemental Methods for references). 0 f the 42 amino acids mutated in Alagille syndrome, 20 are cysteine residues and none are serine residues; few occur in proximity to Ser577 and Ser650. (C) A schematic of 3 canonical EGF-like repeats is shown, along with 2 proposed models for JAG1 EGF-like repeats 9-11. In model 1, residues 562-585 are split between EGFlike repeats 9 and 10 , including a potential disulfide bridge between residues 567 and 572 preceding the start of repeat 10 . Model 1 requires 2 insertions, 1 before and 1 within repeat 10 . In model 2, residues 562 to 585 are included as a single insertion in the second loop of EGF-like repeat 10. (D) 3D modeling shows that Ser577 and Ser650 may reside on the same surface of the JAG1 extracellular domain (model 2).

(gnomAD). Dominant mutations in JAG1 have previously been shown to cause Alagille syndrome, which is characterized by defects in bile ducts, heart, kidney, facies, and vertebrae $(4$, 5). There was no history of cardiac, kidney, or liver disease in affected individuals in either CMT2 family nor evidence of jaundice, xanthomas, or facial dysmorphology. Family 1 individual IV.2 required spine surgery for scoliosis (Figure 1E), but there was no report of vertebral malformations. Detailed clinical case histories are provided in the supplemental material.

The 2 CMT2-associated JAG1 variants both resulted in missense substitutions of serine residues (p.Ser577Arg, family 1; p.Ser650Pro, family 2) within highly conserved regions of the JAG1 extracellular domain (Figure 2, A and B). Both substitutions are predicted to be deleterious by in silico analyses 
A
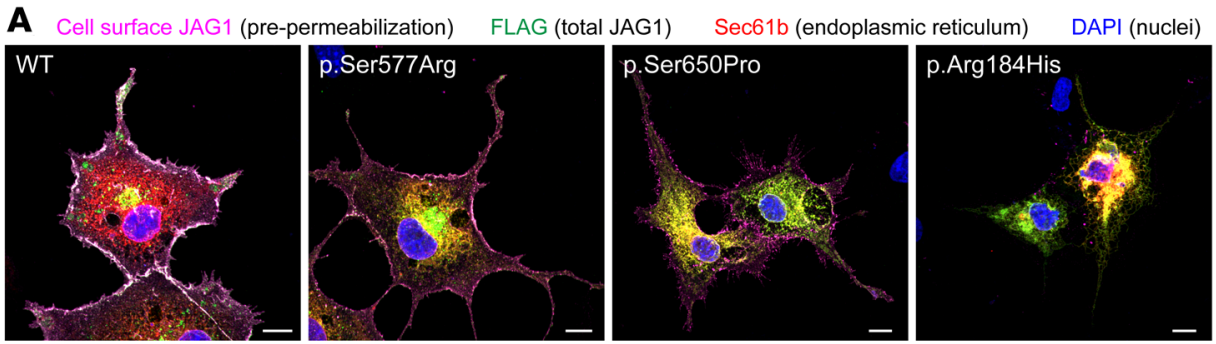

B HEK293T cells

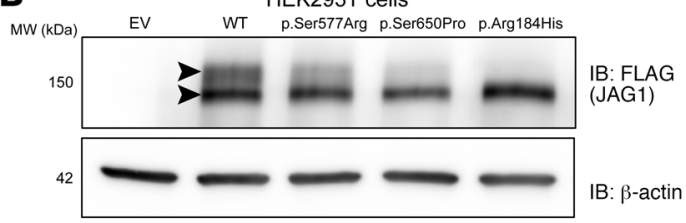

D

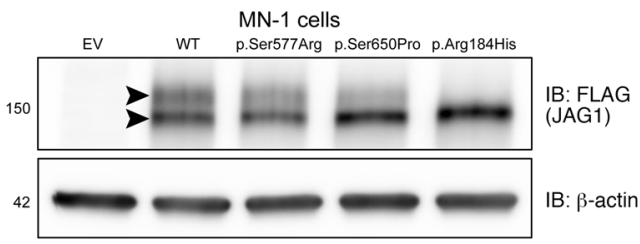

$\mathbf{F}$

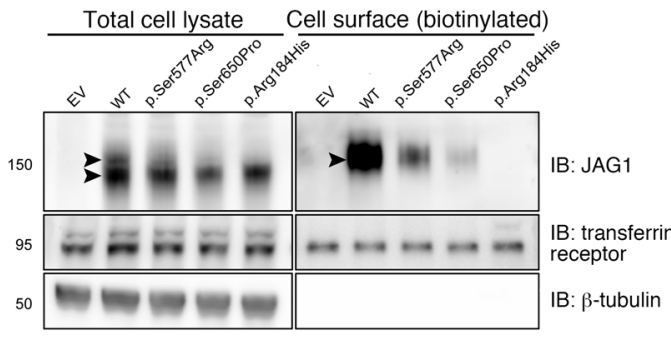

H

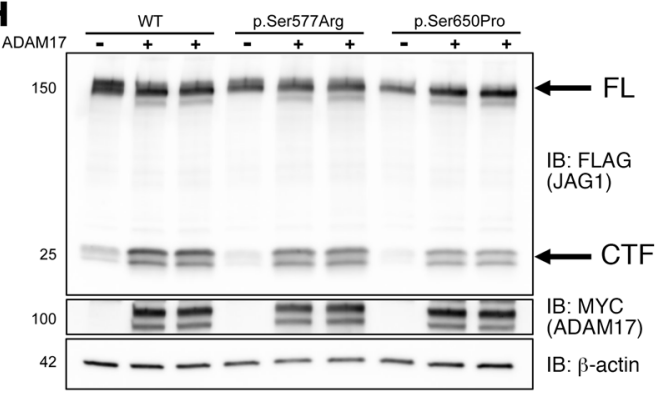

C

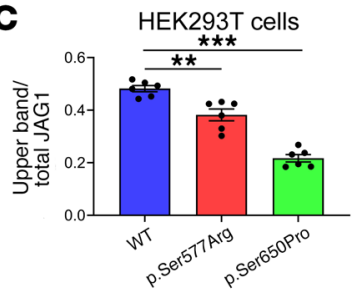

E

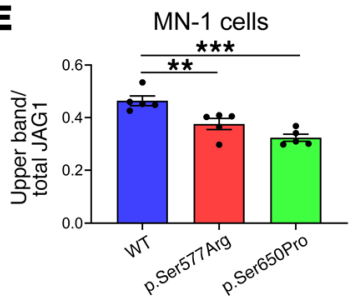

$\mathbf{G}$

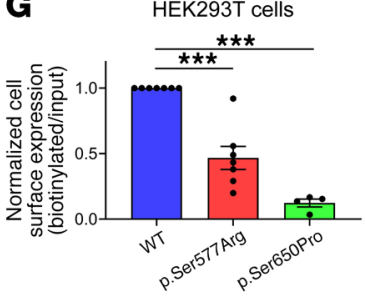

I

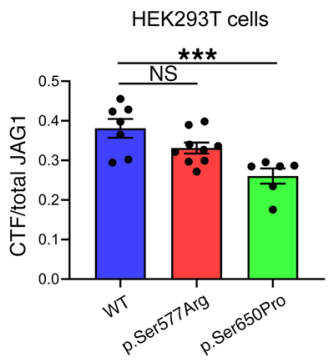

Figure 3. Neuropathy-associated mutations alter JAG1 cell surface expression and glycosylation. (A) Representative images of WT and mutant JAG1-FLAG immunolocalization in transfected COS-7 cells. Scale bars: $10 \mu \mathrm{m}$. (B-E) Representative Western blots of whole cell lysates from HEK293T (B) and MN-1 (D) cells transfected with WT or mutant JAG1-FLAG. Arrowheads indicate the complex (upper band) and simple (lower band) glycosylated forms of JAG1. (C and E) Densitometry-based quantification of the proportion of total JAC1 represented by the higher molecular weight band (C, HEK293T, $n=6$ per condition; $\mathbf{E}, \mathrm{MN}-1, n=5$ per condition). ${ }^{* *} P<0.01 ;{ }^{* *} P<0.001$, Dunnett's test. EV, empty vector. (F-G) Representative Western blot $(\mathbf{F})$ and quantification $(\mathbf{G})$ of JAG1 cell surface biotinylation assays. $n=7$ for WT and p.Ser577Arg; $n=4$ for p.Ser650Pro. ${ }^{* *} P<0.001,1$-sample $t$ test. (H-I) Western blot analysis $(\mathbf{H})$ and quantification (I) of the ADAM17-cleaved C-terminal fragment (CTF) of JAG1, a reflection of the amount of full-length (FL) JAG1 trafficked to the cell surface (11). $n=7$ for WT; $n=9$ for $p$.Ser577Arg; $n=6$ for $p$.Ser650Pro. ${ }^{* *} P<0.001$ by Dunnett's test.
(Supplemental Table 3). Whereas Ser650 is situated within a known domain (EGF-like repeat 11), Ser577 is located within a region (residues 562-585) that has traditionally been annotated as residing between EGF-like repeats 9 and 10 (ref. 4 and Figure $2 \mathrm{~B}$ ). Notably, this region also contains 3 cysteine residues, raising the possibility that it represents a shorter noncanonical EGF-like repeat and thereby contributes to disulfide bridges affecting the structure of the extracellular domain. Although the majority of Alagille syndrome-causing JAG1 mutations are truncating or nonsense mutations, a minority are missense mutations. These missense mutations occur primarily in JAG1 domains that interact directly with Notch receptors (C2 and DSL domains, EGF-like repeats 1-3; ref. 6), the signal peptide, or at cysteine residues thought to contribute to disulfide bridges (Figure $2 \mathrm{~B}$ ). Since there is no experimentally determined structure of the region of JAG1 in which Ser577 and Ser650 reside (6), we performed structural modeling to predict how this region might interact with the surrounding EGF-like repeats and generated 2 possible models (Figure 2C). While model 1 follows current database annotations (Supplemental Figure 1), model 2 is similarly plausible. Interestingly, in model 2, Ser577 and Ser650 are located on the same surface of the protein (Figure 2D), raising the possibility that their mutation could disrupt a ligand interaction with this portion of the extracellular domain or comparably alter the mechanical forces required to activate Notch receptors during JAG1-Notch interactions (7). 

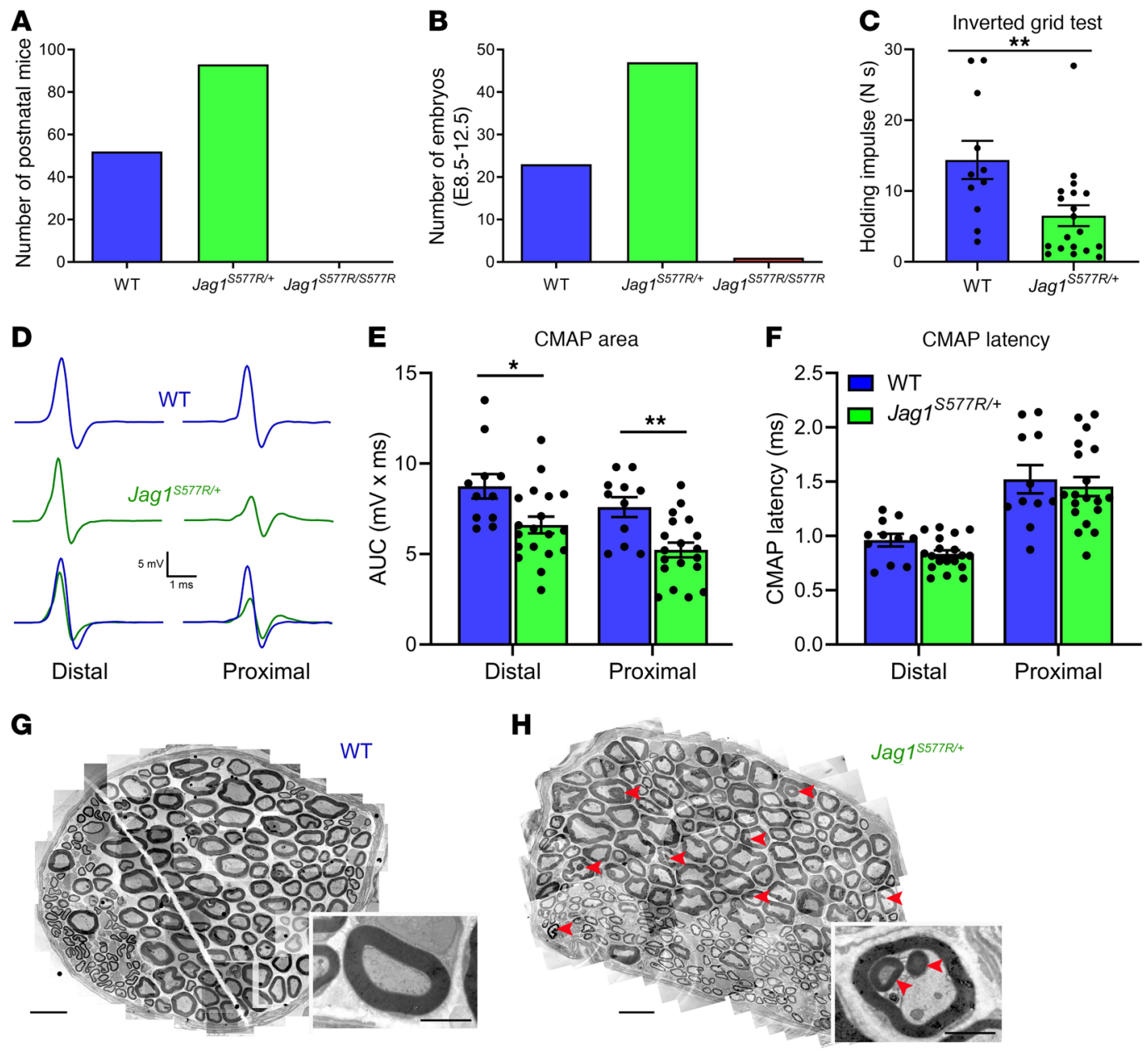

I

J
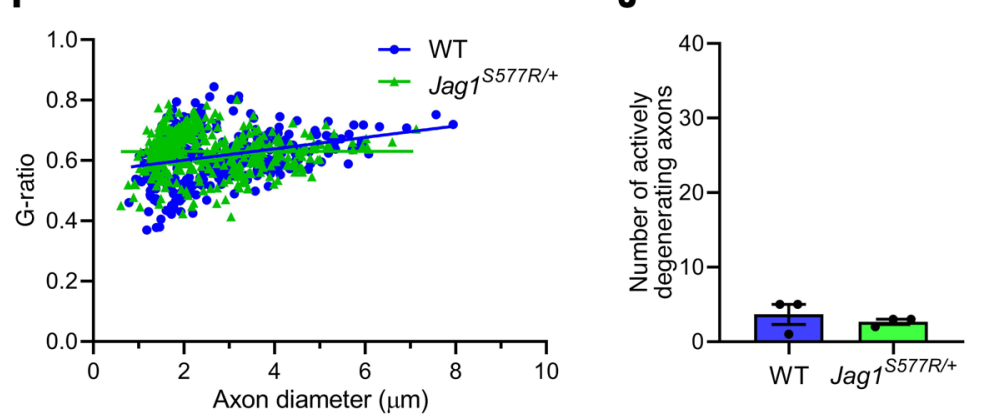

$\mathbf{K}$

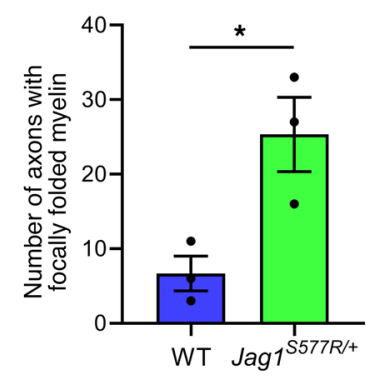

Figure 4. Homozygous expression of the JAG $1^{5577 R}$ mutation causes embryonic lethality in mice, while heterozygous expression results in mild peripheral neuropathy. (A and B) Genotyping of postnatal (A) and embryonic (B; E8.5-E12.5) mice generated from heterozygous intercrosses demonstrates embryonic lethality in Jag $7^{5577 / 5577 R}$ mice. (C-F) Jag $7^{1577 R /+}$ mice display impaired performance in the inverted grid test (C) as well as reductions in CMAP area (D and $\mathbf{E}$ ), but not latency (F). Representative CMAP traces are shown in $\mathbf{D} . n=11$ for WT; $n=19$ for Jag $11^{577 R /+}$. ${ }^{*} P<0.05$; ${ }^{*} P<0.01$, 2-tailed Student's $t$ test. (G and $\mathbf{H})$ TEM reconstructions of the recurrent laryngeal nerve of WT $(\mathbf{G})$ and $J a g 1^{5577 R /+}(\mathbf{H})$ mice. Insets show higher magnification images of individual myelinated axons. Scale bars: $10 \mu \mathrm{m} ; 2 \mu \mathrm{m}$ (insets). Arrowheads indicate regions of focally folded myelin (15). (I-K) Myelinated axons in the recurrent laryngeal nerve of $\mathrm{Jag}^{15777 /+}$ mice exhibit normal g-ratios (I) and numbers of actively degenerating axons (J), but an increased incidence of focally folded myelin (K) For I, $n=350$ axons from 3 WT mice; $n=410$ axons from 3 Jag $7^{5577 /++}$ mice. For J and $\mathbf{K}, n=3$ for WT and Jag ${ }^{5577 R /+}$. ${ }^{*} P<0.05,2$-tailed Student's $t$ test.

To investigate the consequences of the p.Ser577Arg and p.Ser650Pro mutations on JAG1, we performed a series of in vitro studies (Figure 3). Immunocytochemical analysis of transiently transfected COS-7 cells exposed to an anti-JAG1 extracellular domain antibody prior to permeabilization and staining for total JAG1 demonstrated expression of WT JAG1 (JAG1 ${ }^{\mathrm{WT}}$ ) primarily at the plasma membrane (Figure 3A and Supplemental Figure 2). $\mathrm{JAG}^{\text {Ser577Arg }}$ and JAG1 ${ }^{\text {Ser650Pro }}$ were also present at the plasma membrane, but both mutants also exhibited significant immunolocalization with the ER marker Sec61b, suggesting partial retention in the ER and reduced cell surface expression relative to WT (Figure $3 \mathrm{~A}$ and Supplemental Figure 2). In contrast, the well-characterized 
Alagille syndrome-causing mutant JAG1 ${ }^{\text {Arg184His }}$ localized solely to the ER (Figure 3A, Supplemental Figure 2, and refs. 8, 9).

The extracellular domains of Notch receptors and ligands, including JAG1, are extensively posttranslationally modified by glycosylation within the ER (simple glycosylation) and Golgi apparatus (complex glycosylation) (10). As previous studies have shown that only JAG1 protein that has undergone complex glycosylation is trafficked to the cell surface (9), we assessed the glycosylation profiles of the 2 CMT2-associated mutants by Western blot analysis. In whole cell lysates from HEK293T and MN-1 (a motor neuron-like cell line) cells, JAG $1^{\mathrm{WT}}$ migrated as a doublet in the 140 to $170 \mathrm{kDa}$ range, with approximately equivalent expression of the 2 bands (Figure 3, B-E, and ref. 9). The higher and lower molecular weight bands represent complex and simple glycosylated forms of JAG1, respectively, based on differential sensitivity to deglycosylation treatments (refs. 8 and 9 and Supplemental Figure 3, A and B). While JAG1 ${ }^{\text {Ser577Arg }}$ and JAG $1^{\text {Ser650Pro }}$ also migrated as doublets, the complex glycosylated form represented a significantly reduced portion of the total protein for both mutants (Figure 3, B-E). Consistent with the reduction in complex glycosylation, cell surface biotinylation experiments in HEK293T cells demonstrated significantly reduced, but not absent, plasma membrane expression of the 2 mutants (Figure 3, F and G). Assessments of JAG1 cleavage at the plasma membrane by ADAM17 further support reduced cell surface expression of the mutant proteins (ref. 11 and Figure $3, \mathrm{H}$ and I). In contrast, the Alagille syndrome-causing JAG1 ${ }^{\text {Arg184His }}$ mutant migrates as a single band representing the simple glycosylated form (Figure 3, B-E, and Supplemental Figure 3, A and B) and is not detectable in the cell surface fraction (Figure 3, F and G). Together, these data demonstrate that both neuropathy-associated mutant forms of JAG1 are expressed at the cell surface, but at significantly lower levels than WT protein.

Given that Notch signaling outcomes are highly context and cell type dependent $(12,13)$, we adopted an in vivo approach to explore the cellular consequences of the CMT2-associated mutations, using CRISPR/Cas9 gene editing to knock the p.Ser577Arg mutation into the mouse Jag1 gene (C57BL/6J background; Supplemental Figure 4, A and B). The JAG1 proteins of humans and mice exhibit high homology (96.6\% shared sequence identity), including the S577 residue (Figure 2A and Supplemental Figure 5). Past attempts to generate mouse models of Alagille syndrome have demonstrated few relevant phenotypes in mice heterozygous for a JAG1 null allele (Jag1 ${ }^{\text {tmlGrid/+)})}$ and lethality by E10.5 in homozygous mice (5). Mice homozygous for a loss-of-function allele ( $\mathrm{Jag} 1^{\mathrm{Ndr} / \mathrm{Ndr}}$ ) on a $\mathrm{C} 3 \mathrm{H} / \mathrm{C} 57 \mathrm{BL} 6$ background exhibit partial embryonic lethality, with surviving mice exhibiting liver, heart, and kidney defects and craniofacial dysmorphology, consistent with Alagille syndrome (14). As these findings suggest species-specific differences in sensitivity to alterations in JAG1 gene dosage, we established heterozygous intercrosses of the Jag1 $1^{S 577 R}$ mouse line to generate both heterozygous $\left(\operatorname{Jag}^{1577 / \mathrm{R} /}\right)$ and homozygous $\left(\mathrm{Jag} 1^{\text {S577R/S577R }}\right)$ progeny. Genotyping of more than 100 pups generated from these crosses identified no Jag1 ${ }^{\text {S577R/S577R }}$ mice, whereas WT and Jag1 ${ }^{\text {S577R/+ }}$ pups were born at a Mendelian ratio of approximately 1 to 2 (Figure 4A and Supplemental Table 4). Examination of over 70 embryos at gestational ages E8.5 to E12.5 revealed only a single living Jag1 $1^{5577 R / S 577 R}$ embryo (Figure 4B). In addition, several dead Jag1 ${ }^{\text {S577R/S577R }}$ embryos were observed at E8.5 (but not at later time points), confirming a clear functional relevance of the p.Ser577Arg substitution and suggesting that embryonic lethality primarily occurs immediately prior to E8.5. Motor behavioral assessments of Jag1 ${ }^{S 577 R /+}$ mice (16-20 months of age) revealed impaired performance in the inverted grid test of limb strength relative to WT littermates (Figure 4C), but normal performance in the accelerating rotarod assay (Supplemental Figure 4D). Jag1 $1^{5577 R /+}$ mice (19-23 months of age) also exhibited reductions in compound muscle action potential (CMAP) area in assessments of peripheral nerve electrophysiology (Figure 4, D and $\mathrm{E})$, with a slight, but nonsignificant $(P=0.054$; by 2-tailed Student's $t$ test), reduction in CMAP distal latency (Figure 4F). Morphological assessments of the recurrent laryngeal nerve in Jag1 ${ }^{S 577 R /+}$ mice at 19 to 23 months of age (Figure 4, G-K) did not reveal alterations of axon or myelin sheath diameter (g-ratio; Figure 4I), numbers of actively degenerating axons (Figure $4 \mathrm{~J}$ ), total myelinated axon number (Supplemental Figure 4E), or neuromuscular junction innervation (Supplemental Figure 4, F and G) at this time point, but did demonstrate an increased frequency of focally folded myelin (ref. 15 and Figure 4K). This peripheral nerve abnormality has been observed with aging (16), several forms of hereditary neuropathy (15), and experimental disruptions of axon-glial interactions (17, 18). Together, these findings indicate that heterozygous expression of the p.Ser577Arg mutation causes a mild peripheral neuropathy in mice. The severe and mild phenotypes observed with homozygous and heterozygous expression of the mutant Jag1 allele, respectively, are similar to gene dosage effects observed in mouse models of other autosomal dominant forms of axonal neuropathy (19-22).

Together, our clinical, genetic, biochemical, and mouse model findings implicate JAG1 mutations as a cause of CMT2 with severe vocal fold paresis. These results broaden the spectrum of diseases associated with alterations in the Notch pathway and point to a key role for JAG1 in peripheral nerve, particularly the recurrent laryngeal nerve. The absence of Alagille syndrome phenotypes in both CMT2 families suggests that contrasting disease mechanisms underlie the 2 disorders. The vast majority of Alagille syndrome-causing mutations are JAG1 gene deletions or truncating mutations, suggesting that haploinsufficiency represents the primary disease mechanism $(4,5)$. The identification of Alagille syndrome-causing mutations in $\mathrm{NOTCH} 2$ further points to a key role of reduced JAG1/NOTCH2 signaling in disease pathogenesis $(4,5)$. The specific involvement of peripheral nerve in CMT2 suggests that p.Ser577Arg and p.Ser650Pro may precipitate disease through a more complex, or tissue-specific, mechanism or mechanisms, potentially involving specific reductions or alterations in Notch receptor trans-activation and/or cis-inhibition. Within the peripheral nervous system, JAG1 is expressed by both axons and Schwann cells, whereas Notch receptor expression appears to occur principally in Schwann cells $(23,24)$. Elevated expression of JAG1 has also been described in reactive spinal cord astrocytes in a mouse model of the inherited neurodegenerative disease spinal muscular atrophy (25). While Notch signaling has been shown to control several important aspects of peripheral nerve development and plasticity (23), the specific roles played by JAG1 in the peripheral nervous system remain to be determined. Continued elucidation of these roles will be central to identifying potential pathological and pathophysiological alterations in peripheral 
nerve that may be associated with pan-Notch- and JAG1-targeted therapeutics and determining how these may best be evaluated in preclinical and clinical settings.

\section{Methods}

Detailed experimental methods are included with the Supplemental Methods. Specific statistical tests employed and sample sizes are described in each figure legend. All error bars represent SEM.

Study approval. IRB approval was obtained from the NIH and St. George's University London. Written informed consent was obtained from all subjects, including for the use of the photographs shown in Figure 1E. All animal procedures followed NIH guidelines and were approved by the IACUCs at Johns Hopkins University School of Medicine and the Jackson Laboratory.

\section{Author contributions}

CJS, AHC, and BJT directed the study. JMS, WWM, and CJS wrote and edited the manuscript with AHC and RG. CJS, MHM, LAH, MMM, ELB, and HM evaluated patients and clinical data. JOJ and KESB carried out the gene sequencing and analysis. Haplotype analysis was performed by JOJ. In vitro studies were completed by JMS and WHA. Structural modeling was performed by RG. The Jag $1^{5577 R}$ mouse line was generated by ARZ and CML and characterized by JMS, LK, JSH, and PCSR. Peripheral nerve electrophysi- ology was performed by KLM and MHF. The order of the 2 co-first authors was determined based on the scope of their respective contributions.

\section{Acknowledgments}

We thank the families for their participation in this study, Kenneth (Kurt) Fischbeck and Christy Ludlow for their help enrolling family 1, and Minoru Tada for providing JAG1 plasmids. This work was supported by NIH-National Institute of Neurological Disorders and Stroke (NIH-NINDS) R01NS062869, the Intramural Research Program of the NIH, NINDS intramural research funds, the National Institute on Aging (Z01-AG000949-02), the Medical Research Council (G1002279), and The Jackson Laboratory's Genetic Engineering Technologies Scientific Service. WWM was supported in part by NIH-NINDS R25NS065729 and WHA by NIH-NINDS F31NS105404.

Address correspondence to: Charlotte J. Sumner, Rangos Building, Room 234, 855 N. Wolfe St., Baltimore, Maryland 21205, USA. Phone: 410.502.6085; Email: csumner1@jhmi.edu. Or to: Andrew H. Crosby, University of Exeter Medical School, RILD Wellcome Wolfson Centre, Royal Devon and Exeter NHS Foundation Trust, Barrack Road, Exeter, X2 5DW United Kingdom. Phone: 44.1392.408302; Email: a.h.crosby@exeter.ac.uk.
1. Braune EB, Lendahl U. Notch -- a Goldilocks signaling pathway in disease and cancer therapy. Discov Med. 2016;21(115):189-196.

2. Lafkas D, et al. Therapeutic antibodies reveal Notch control of transdifferentiation in the adult lung. Nature. 2015;528(7580):127-131.

3. Weis J, Brandner S, Lammens M, Sommer C, Vallat JM. Processing of nerve biopsies: a practical guide for neuropathologists. Clin Neuropathol. 2012;31(1):7-23.

4. Grochowski CM, Loomes KM, Spinner NB. Jagged1 (JAG1): Structure, expression, and disease associations. Gene. 2016;576(1 Pt 3):381-384.

5. Gilbert MA, Spinner NB. Alagille syndrome: genetics and functional models. Curr Pathobiol Rep. 2017;5(3):233-241.

6. Luca VC, et al. Notch-Jagged complex structure implicates a catch bond in tuning ligand sensitivity. Science. 2017;355(6331):1320-1324.

7. Wang X, Ha T. Defining single molecular forces required to activate integrin and notch signaling. Science. 2013;340(6135):991-994.

8. Morrissette JD, Colliton RP, Spinner NB. Defective intracellular transport and processing of JAG1 missense mutations in Alagille syndrome. Hum Mol Genet. 2001;10(4):405-413.

9. Tada M, Itoh S, Ishii-Watabe A, Suzuki T, Kawasaki N. Functional analysis of the Notch ligand Jagged1 missense mutant proteins underlying Alagille syndrome. FEBS J. 2012;279(12):2096-2107.
10. Harvey BM, Haltiwanger RS. Regulation of Notch function by O-glycosylation. Adv Exp Med Biol. 2018;1066:59-78.

11. Parr-Sturgess CA, Rushton DJ, Parkin ET. Ectodomain shedding of the Notch ligand Jagged 1 is mediated by ADAM17, but is not a lipid-raftassociated event. Biochem J. 2010;432(2):283-294.

12. Nandagopal N, Santat LA, LeBon L, Sprinzak D, Bronner ME, Elowitz MB. Dynamic ligand discrimination in the Notch signaling pathway. Cell. 2018;172(4):869-880.e19.

13. Gama-Norton L, et al. Notch signal strength controls cell fate in the haemogenic endothelium. Nat Commun. 2015;6:8510.

14. Andersson ER, et al. Mouse model of Alagille syndrome and mechanisms of Jagged 1 missense mutations. Gastroenterology. 2018;154(4):1080-1095.

15. Weis J, et al. Towards a functional pathology of hereditary neuropathies. Acta Neuropathol. 2017;133(4):493-515.

16. Ceballos D, Cuadras J, Verdú E, Navarro X. Morphometric and ultrastructural changes with ageing in mouse peripheral nerve. J Anat. 1999;195(Pt 4):563-576.

17. Golan N, et al. Genetic deletion of Cadm 4 results in myelin abnormalities resembling Charcot-Marie-Tooth neuropathy. J Neurosci. 2013;33(27):10950-10961.

18. Sheean ME, et al. Activation of MAPK overrides the termination of myelin growth and replaces Nrg1/ErbB3 signals during Schwann cell development and myelination. Genes Dev. 2014;28(3):290-303.

19. Detmer SA, Vande Velde C, Cleveland DW, Chan DC. Hindlimb gait defects due to motor axon loss and reduced distal muscles in a transgenic mouse model of Charcot-Marie-Tooth type 2A. Hum Mol Genet. 2008;17(3):367-375.

20. Cartoni R, et al. Expression of mitofusin 2(R94Q) in a transgenic mouse leads to Charcot-MarieTooth neuropathy type 2A. Brain. 2010;133(Pt 5): 1460-1469.

21. Strickland AV, et al. Characterization of the mitofusin $2 \mathrm{R} 94 \mathrm{~W}$ mutation in a knock-in mouse model. J Peripher Nerv Syst. 2014;19(2):152-164.

22. Nandini S, Conley Calderon JL, Sabblah TT, Love R, King LE, King SJ. Mice with an autosomal dominant Charcot-Marie-Tooth type $2 \mathrm{O}$ disease mutation in both dynein alleles display severe moto-sensory phenotypes. Sci Rep. 2019;9(1):11979.

23. Woodhoo A, et al. Notch controls embryonic Schwann cell differentiation, postnatal myelination and adult plasticity. Nat Neurosci. 2009;12(7):839-847.

24. $\mathrm{Hu} \mathrm{X}$, et al. BACE1 regulates the proliferation and cellular functions of Schwann cells. Glia. 2017;65(5):712-726.

25. Caraballo-Miralles V, et al. Notch signaling pathway is activated in motoneurons of spinal muscular atrophy. Int J Mol Sci. 2013;14(6):11424-11437. 